\title{
Attempt to Quit Tobacco Smoking among Male Smokers in Vietnam and Predictive Factors: Findings from Global Adult Tobacco Survey (GATS)
}

\author{
Giang Bao Kim, Diep Bich Pham¹, Hai Thi Phan², Minh Dai Le ${ }^{3}$, Minh Van \\ Hoang $^{4}$
}

\begin{abstract}
Background: Vietnam is among countries with highest prevalence of tobacco smoking, attempt to quit is an important indicator to monitor the effectiveness of tobacco control efforts. This paper aims to describe smoking quit attempt and examine its association with some individual characteristics among male smokers. Methods: Data from the Global Adult Tobacco Survey in Vietnam in 2015 was analyzed for a sample of 1,903 male smokers taking from the national representative sample of 8,996 adults aged 15 years and above. Results: Proportion of quit attempt during 12 months prior to the interview among male smoker was $37.1 \%$. Attempt to quit smoking was significantly associated with age (OR=2.84 and 95\% CI: 1.43-5.66 for those aged 55 years and older vs. those aged 24 years and younger), with knowledge of harmful effects on health (OR=1.97 and 95\% CI: 1.45-2.66 for those who could list 6 to 7 diseases vs. those who could list 3 or less diseases), number of channels with anti smoking message (OR=1.72 and $95 \%$ CI: 1.21-2.45 for those who had exposure from 3 channels or more vs. those who did not expose any channels), number of years smoking $(\mathrm{OR}=0.59$ and $\mathrm{OR}=0.40$ for those with less than 15 years smoking vs. those with 25 to 34 years smoking and more than 35 years smoking, respectively). Conclusion: Intervention to improve knowledge of tobacco harmful effects, and access to multiple and modern antismoking communication channels would be effective to raise quit attempt among smokers. Research to promote effectiveness of quit advice by health staff should be paid more attention.
\end{abstract}

Keywords: attempt to quit- tobacco smoking- tobacco cessation- Global Adult Tobacco Survey- Vietnam

Asian Pac J Cancer Prev, 22 (7), 2061-2067

\section{Introduction}

Globally, tobacco use is currently responsible for the deaths of about six million people across the world each year (World Health Organization, 2011). It is predicted that by 2030 smoking will kill more than 8 million people each year, and more than $80 \%$ of which will be in low and middle income countries if the current pattern persists (World Health Organization, 2008, 2011).

The Global Action Plan for the Prevention and Control of Non communicable Diseases during the period 2020 and 2030 identifies that $30 \%$ relative reduction of tobacco use in persons aged 15 years and above is among nine key targets to help prevent premature avoidable mortality from Non communicable diseases (World Health Organization, 2013). In order to assist tobacco control policies worldwide, WHO introduced a package of six evidence-based tobacco control demand reduction measures that are proven to reduce tobacco use in 2008, known as MPOWER package measures. Offer to quit smoking is an important component of tobacco control interventions at country level to reduce the demand for tobacco (World Health Organization, 2008). Health staff is expected to ask patients about tobacco use status and give advice to stop smoking if a patient reported using tobacco. Available evidences indicate that the brief advice to quit from a physician could improve cessation rates and is highly cost-effective (Stephen Babb et al., 2017). However, not all smokers got advice to quit smoking. In the United State of America, only 57.2\% of adult smokers who had seen a health professional in the past year reported receiving advice to quit (Department of Health and Human Services, 2020).

Attempt to quit smoking varied across ages, socio-economc groups. It is more likely to happen among smokers with a higher level of nicotine dependence, more negative to smoking behavior (Eleni Vangeli et al., 2011; Hyland et al., 2016), who have higher level of motivation,

${ }^{I}$ School of Preventive Medicine and Public Health, Hanoi Medical University, Hanoi, Vietnam. ${ }^{2}$ Viet Nam Tobacco Control Fund, MOH Viet Nam, Hanoi, Vietnam. ${ }^{3}$ Medical English Club, Hanoi Medical University, Hanoi, Vietnam. ${ }^{4}$ Hanoi University of Public Health (HUPH), Hanoi, Vietnam.*For Correspondence: kimbaogiang@hmu.edu.vn 
who had higher level of confidence in quitting, who had previous quit attempt (Eleni Vangeli et al., 2011), who loose smoking friends over period of time (Bethany et al., 2016). Inversely, those who have greater enjoyment with smoking (Eleni Vangeli et al., 2011; Hitchman et al., 2014) and patients who are "disinterest in receiving advice" (Medawela et al., 2021) are less likely to make quit attempt. The relationship between age and education are not consistent between studies (Eleni Vangeli et al., 2011; Hitchman et al., 2014; Hyland et al., 2016)

Vietnam is among countries with highest prevalence of tobacco smoking. In 2010, the smoking prevalence among adults aged 15 years and above was $23.8 \%$, and that was much higher among males (47.4\%). Meanwhile, tobacco cessation was not well developed. According to the Global Adult Tobacco Survey (GATS) in 2010, only $29.7 \%$ of smokers got advice to quit by a health care provider in the a 12-month period (Ministry of Health of Viet Nam et al., 2010). In 2010, among male smokers, only $19.1 \%$ quitted smoking; $55.6 \%$ attempted to quit and $29.6 \%$ intended to quit smoking. The Law on Prevention and Control of Tobacco Harms in Vietnam in 2012 points out that tobacco cessation is an important measure to reduce smoking prevalence in Vietnam (National Assembly of Vietnam, 2012). Since then, Vietnam started to build and enhance cessation services and tobacco cessation counselling. In 2015, tobacco cessation counselling unit at a central hospital was established and several training activities have been conducted for 60 resource trainers and more than 600 health staffs at 63 provinces to prepare for the expansion of this service in other hospitals and health facilities (Vietnam Tobacco Control Fund, 2015).

The Global Adult Tobacco Survey in 2015 (GATS 2015) in Vietnam is the second round of GATS in Vietnam after the first survey in 2010. The GATS aims to provide information to monitor tobacco control indicators in Vietnam according to MPOWER measures. In this second survey, tobacco cessation was also investigated. Updates on tobacco cessation indicators and its' associated factors is valuable to plan for effective tobacco control activities. This paper used data from GATS 2015 in Vietnam to describe smoking quit attempts and association between quit attempt and socioeconomic and demographic factors among male smokers.

\section{Materials and Methods}

\section{Data source}

This paper used data from GATS 2015 in Vietnam. GATS is an international survey that was initiated by the Center for Diseases Control and the World Health Organization and financially supported by Bloomberg. In Vietnam, the survey was conducted by Ministry of Health, Hanoi Medical University, the General Statistics Office and with technical support from World Health Organization and Center for Diseases Control. The target population for this survey includes adults aged 15 years old or older in Vietnam. The sample of 9,513 households $(4,725$ in urban areas and 4,788 in rural areas) was calculated to obtain reliable estimates of the key variables of gender and urban or rural areas and covers $15 \%$ of total population. In each household, an adult aged 15 years old or older was randomly selected for the interview. The completed sample was 8,996 persons, yielding a response rate of $95.8 \%$. Data collection was conducted by the General Statistics Office of Vietnam under technical supports of World Health Organization, Hanoi Medical University, Vietnam Steering Committee on Smoking and Health and Center for Disease Control and Prevention. An international standardized questionnaire was adapted through several steps of back translation procedures. The details descriptions was presented elsewhere (Ministry of Health of Viet Nam et al., 2016). Among 8,996 adults who completed the interviews, 1,972 persons reported as smokers in which 1,903 smokers were male. Thus, the sample used for this paper is 1,903 that equivalent to an weighted number of 15,213,471 male smokers according to Vietnamese population in 2015.

\section{Variables used}

Dependent variables were "attempt to quit smoking during the last 12 months": smokers who reported at least one attempt to quit smoking during the last 12 months prior to the interviews. This can be any actions as the effort to stop smoking, such as joining direct advice session for cessation; using Nicotine replacement therapy; using regular chewing gum without any nicotine; using other prescription medications (for example, Bupropion, Varenicline), using traditional medicines; calling quit line or a smoking telephone support line; switching to smokeless tobacco; trying to quit without assistance.

Independent variables included (1) age groups; (2) education level, (3) occupation, (4) Wealth quintile based on household's assets, (5) Residence (urban, rural); (6) Knowledge of diseases caused by tobacco smoking; (7) health staff 's advice to quit smoking when visiting health facilities during the last 12 months; (8) Number of communication channels for anti-smoking message during the 30 days prior to the interview; (9) Notice of health warning in cigarette package during the 30 days prior to the interview; (10) Number of years smoking; (11) Time after waking up for the first cigarettes (tobacco dependent levels).

\section{Data Analysis}

Descriptive statistics were performed to estimate frequencies and percentages for dependent and independent variables of interest. Prevalence of attempt to quit tobacco smoking was estimated for different groups based on individual characteristics. Logistic regression analyses were employed to examine the association between attempt to quit smoking and other factors among male smokers. Weight value were identified for each case in the database using following steps as described elsewhere (Global Adult Tobacco Survey Collaborative Group, 2020): "1) Determine the base weight to account for all steps of random selection that led to the sample of population members; 2) Adjust for nonresponse to compensate for sample imbalance due to differential success in sample recruitment; 3) Further adjust for incomplete frame coverage to adjust for imbalance due to a sampling frame that does not fully cover the population 
targeted for study; and 4. Further adjust to calibrate the final set of adjusted weights to the distribution of the population by characteristics that are highly correlated with the key study outcome measures (i.e., tobacco use behavior in GATS)". Weights were used in all analyses, adjusted odds ratios (ORs) were estimated with 95\% confidence intervals (CIs).

\section{Ethical considerations}

This survey complied with standard procedures that internationally used. It was approved by Ministry of Health of Vietnam, World Health Organization, Center for Disease Control and Prevention, and local authorities. Informed consent was gathered from respondent by the time of the interview.

\section{Results}

The proportion of male smokers at the age of 35 to 44 years old was highest (27\%). The youngest group (less than 25 years old) was accounted for only $12.7 \%$. More than half of male smokers had primary and secondary level of education (54.8\%). Those had secondary and higher levels of education accounted for $15.7 \%$. Self-employees accounted for $73.2 \%$, following by employees of government and non-government organizations that accounted for $17.1 \%$. Regarding knowledge of harmful effects on health of tobacco smoking, $48.1 \%$ male smokers could list only 3 or less diseases caused by tobacco. Of $68.8 \%$ male smokers lived in rural areas.

During the last 12 months $11.9 \%$ got advice to quit tobacco smoking from health staff. During a 30 day-period prior to the interview, the proportions of male smokers did not receive the anti-smoking message from any media channels was $24.2 \%$, received from 1-2 channels was $47.1 \%$, from 3 channels and more was $28.7 \%$.

Among smokers, one third of them smoked less than 70 cigarettes per week $(33.0 \%)$, one third of them smoked from 70 to less than 140 cigarettes $(33.3 \%)$ per week and the other one third of them smoked 140 cigarettes per week (33.7\%). 30.5\% smokers had smoked for less than 15 years, $21.3 \%$ smoked tobacco for 35 years. More than half $(53.6 \%)$ of smokers needed to smoke the first cigarette just 30 minutes after waking up.

As shown in Table 3 , the overall proportion of attempt to quit tobacco smoking among male smokers in Vietnam during 12 months prior to the interview was $37.1 \%$. The proportion of attempt to quit smoking among male smokers at the oldest age group was highest at $40.3 \%$ and this was lowest at the youngest age group $(34.9 \%)$ and those aged from 35 to 44 years $(34.5 \%)$. The proportion of attempt to quit was highest among those male smokers with upper secondary education level (49\%) and was lowest among those with lowest level of education (below primary: 29.6\%). Those male smokers with more knowledge on harmful effects of tobacco on health had high rate of attempt to quit tobacco smoking (53.4\% of those who could list 6 to 7 diseases caused by tobacco smoking compared to $32.3 \%$ of those who could list 3 or less diseases). Those who received advice to quit smoking from health staff had higher rate of attempt to quit compared to those who did not receive the advice (45.6\% vs. $35.9 \%$ ). There was a clear tendency that male smokers who received anti-smoking message during the last 30 days from more channels having higher rate of attempt to quit smoking $(45.8 \% ; 36.7 \%$ and $27.4 \%$ for groups had access to 3 channels or more, 1-2 channels; no channel at all, respectively). Those who had noticed health warning message on tobacco package during the last 30

Table 1. Characteristics of the Study Participants (Weighted Numbers and Percentages)

\begin{tabular}{|c|c|c|c|}
\hline \multirow[t]{2}{*}{ Variables } & \multicolumn{2}{|c|}{ Frequency } & \multirow{2}{*}{$\begin{array}{l}\text { Weighted } \\
\text { Percentage }\end{array}$} \\
\hline & Un weighted & Weighted & \\
\hline \multicolumn{4}{|l|}{ Age group } \\
\hline$<=24$ & 118 & $1,798,362$ & 12.4 \\
\hline $25-34$ & 308 & $3,198,218$ & 22.9 \\
\hline $35-44$ & 432 & $3,839,393$ & 27.5 \\
\hline $45-54$ & 468 & $2,690,562$ & 19 \\
\hline$>=55$ & 457 & $2,644,988$ & 18.2 \\
\hline \multicolumn{4}{|l|}{ Educational level } \\
\hline Below primary school & 332 & $2,669,299$ & 17.6 \\
\hline $\begin{array}{l}\text { Primary and basic } \\
\text { secondary schools }\end{array}$ & 1,035 & $8,333,293$ & 54.8 \\
\hline Secondary school & 292 & $2,392,554$ & 15.7 \\
\hline Above secondary school & 243 & $1,812,878$ & 11.9 \\
\hline \multicolumn{4}{|l|}{ Occupation } \\
\hline $\begin{array}{l}\text { Government and non- } \\
\text { government Employee }\end{array}$ & 323 & $2,598,938$ & 17.1 \\
\hline Self-employee & 1,335 & $11,141,704$ & 73.2 \\
\hline $\begin{array}{l}\text { Unemployed } \\
\text { (able and unable to work) }\end{array}$ & 135 & 857,174 & 5.6 \\
\hline $\begin{array}{l}\text { Student, Homemaker, } \\
\text { Retired, others }\end{array}$ & 110 & 615,655 & 4 \\
\hline \multicolumn{4}{|c|}{ Knowledge of harmful effects on health } \\
\hline$<=3$ diseases & 918 & $7,324,903$ & 48.1 \\
\hline $4-5$ diseases & 557 & $4,547,023$ & 29.9 \\
\hline 6-7 diseases & 428 & $3,341,545$ & 22 \\
\hline \multicolumn{4}{|l|}{ Wealth quintiles } \\
\hline Poorest & 386 & $3,118,872$ & 20.5 \\
\hline Second poorest & 527 & $4,155,632$ & 27.3 \\
\hline Averaged & 290 & $2,482,949$ & 16.3 \\
\hline Second richest & 320 & $2,541,471$ & 16.7 \\
\hline Richest & 380 & $2,914,547$ & 19.2 \\
\hline \multicolumn{4}{|l|}{ Residence } \\
\hline Urban & 874 & $4,742,270$ & 31.2 \\
\hline Rural & 1,029 & $10,471,201$ & 68.8 \\
\hline \multicolumn{4}{|l|}{ Advice to quit by health staff } \\
\hline No & 1646 & $13,409,602$ & 88.1 \\
\hline Yes & 257 & $1,803,869$ & 11.9 \\
\hline \multicolumn{4}{|c|}{ Number of channels for anti-smoking message } \\
\hline No & 442 & $3,688,708$ & 24.2 \\
\hline $1-2$ channels & 901 & $7,158,516$ & 47.1 \\
\hline 3 channels and more & 560 & $4,366,247$ & 28.7 \\
\hline \multicolumn{4}{|l|}{ Notice of health warning } \\
\hline No & 168 & $1,302,797$ & 8.6 \\
\hline Yes & 1,735 & $13,910,674$ & 91.4 \\
\hline Total & 1,903 & $15,208,024$ & 100 \\
\hline
\end{tabular}

Asian Pacific Journal of Cancer Prevention, Vol $22 \mathbf{2 0 6 3}$ 
Table 2. Smoking Status of Smokers

\begin{tabular}{lccc}
\hline & Unweight & Weighted & $\begin{array}{c}\text { Weighted } \\
\text { Percentage }\end{array}$ \\
\hline Number of cigarette per week & & & \\
Less than 70 & 599 & $5,023,669$ & 33 \\
From 70 to less than 140 & 642 & $5,069,759$ & 33.3 \\
140 and more & 662 & $5,120,043$ & 33.7 \\
Number of years smoking & & & \\
Less than 15 years & 408 & $4,633,289$ & 30.5 \\
15 -24 years & 446 & $3,839,443$ & 25.2 \\
25 -34 years & 499 & $3,505,730$ & 23 \\
35 years and more & 550 & $3,235,009$ & 21.3 \\
Time after waking up for the first cigarette & \\
Less than 30 minutes & 1,027 & $8,157,275$ & 53.6 \\
30-less than 60 minutes & 315 & $2,470,226$ & 16.2 \\
60 minutes and longer & 300 & $2,370,644$ & 15.6 \\
$\quad$ Non daily smoker & 261 & $2,215,326$ & 14.6 \\
Total & 1,903 & $15,213,471$ & 100 \\
\hline
\end{tabular}

Table 3. Percentage of Attempt to Quit among Male Smokers Aged 15 Years in Vietnam by Socioeconomic Status and Smoking Status

\begin{tabular}{|c|c|c|}
\hline Variables & Weighted \% & $(95 \% \mathrm{CI})$ \\
\hline \multicolumn{3}{|l|}{ Age group } \\
\hline$<=24$ & 34.94 & $34.87-35.00$ \\
\hline $25-34$ & 38.61 & $38.56-38.66$ \\
\hline $35-44$ & 34.46 & $34.42-34.51$ \\
\hline $45-54$ & 37.31 & $37.25-37.37$ \\
\hline$>=55$ & 40.34 & $40.28-40.40$ \\
\hline \multicolumn{3}{|l|}{ Educational level } \\
\hline Below primary & 29.61 & $29.56-29.67$ \\
\hline Primary, basic secondary & 38.79 & $38.75-38.82$ \\
\hline Secondary & 30.53 & $30.47-30.59$ \\
\hline Above secondary & 48.99 & $48.92-49.06$ \\
\hline \multicolumn{3}{|l|}{ Occupation } \\
\hline Employee & 42.06 & $42.00-42.12$ \\
\hline Self-employed & 35.48 & $35.45-35.51$ \\
\hline Unemployed & 40.05 & $39.95-40.15$ \\
\hline Other & 40.9 & $40.78-41.03$ \\
\hline \multicolumn{3}{|l|}{ Wealth quintiles } \\
\hline Poorest & 30.37 & $30.32-30.41$ \\
\hline Second poorest & 39.97 & $39.93-40.02$ \\
\hline Averaged & 39.42 & $39.36-39.47$ \\
\hline Second richest & 42.97 & $42.91-43.02$ \\
\hline Richest & 33.12 & $33.06-33.18$ \\
\hline \multicolumn{3}{|c|}{ Knowledge of harmful effects on health } \\
\hline$<=3$ diseases & 32.35 & $32.31-32.38$ \\
\hline 4-5 diseases & 32.69 & $32.64-32.73$ \\
\hline 6-7 diseases & 53.43 & $53.38-53.48$ \\
\hline \multicolumn{3}{|l|}{ Residence } \\
\hline Urban & 36.94 & $36.90-36.99$ \\
\hline Rural & 37.14 & $37.11-37.17$ \\
\hline
\end{tabular}

Table 3. Continued

\begin{tabular}{|c|c|c|}
\hline Variables & Weighted \% & $(95 \% \mathrm{CI})$ \\
\hline \multicolumn{3}{|l|}{ Advice to quit by health staff } \\
\hline No & 35.94 & $35.91-35.96$ \\
\hline Yes & 45.59 & $45.51-45.66$ \\
\hline \multicolumn{3}{|l|}{ Number of channels } \\
\hline None & 27.42 & $27.38-27.47$ \\
\hline 1-2 channels & 36.74 & $36.70-36.78$ \\
\hline 3 channels and more & 45.79 & $45.75-45.84$ \\
\hline \multicolumn{3}{|l|}{ Notice of health warning } \\
\hline No & 26.01 & $25.93-26.08$ \\
\hline Yes & 38.12 & $38.09-38.14$ \\
\hline \multicolumn{3}{|l|}{ Number of cigrarettes per week } \\
\hline Less than 70 & 39 & $38.96-39.05$ \\
\hline From 70 to less than 140 & 38.93 & $38.89-38.98$ \\
\hline From 140 & 33.36 & $33.32-33.4$ \\
\hline \multicolumn{3}{|l|}{ Number of years smoking } \\
\hline Less than 15 years & 39.09 & $39.04-39.13$ \\
\hline $15-24$ years & 37.14 & $37.09-37.19$ \\
\hline $25-34$ years * & 35.79 & $35.74-35.84$ \\
\hline From 35 years $*$ & 35.53 & $35.48-35.58$ \\
\hline \multicolumn{3}{|c|}{ Time after waking up for the first cigarette } \\
\hline Less than 30 minutes & 32.73 & $32.70-32.77$ \\
\hline 30-less than 60 minutes* & 39.5 & $39.44-39.56$ \\
\hline 60 minutes and longer* & 47.09 & $47.03-47.15$ \\
\hline Non daily smoker & 39.67 & $39.60-39.73$ \\
\hline Overall & 37.08 & $37.05-37.1$ \\
\hline
\end{tabular}

days also had higher rate of attempt to quit as compared to those who did not (38.1\% vs. $26.0 \%$ ). Compared to those who smoked for less than 15 years, those smokers who had longer years of smoking (from 25 years and more) had a slightly lower rate of attempt to quit $(35.8 \%$ and $35.5 \%$ vs. $39.1 \%$ ). Those smokers who were more dependent to tobacco (shorter time after waking up having the first cigarette) had significantly lower rates of attempt to quit smoking: $32.73 \%$ among those smokers who belonged to the group "less than 30 minutes" compared to $39.5 \%$ of the group "from 30 to less than 60 minutes" and $47.09 \%$ of the group "60 minutes and longer".

The logistic regression models only found significant associations between attempt to quit smoking with age, knowledge of harmful effects on health, number of channels for anti smoking message, number of years smoking, and time after waking up for the first cigarette. Those who were 55 years and older 2.84 times more likely to attempt to quit smoking than those at the age group of 24 years and younger (95\% CI: 1.43-5.66). Male smokers who could list 6 to 7 diseases caused by tobacco smoking 1.97 times more likely than those who could 3 or less diseases ( $\mathrm{OR}=1.97$; 95\% CI: 1.45-2.66). Compared to those who did not receive anti-smoking message from any channels, the odds of attempt to quit was statistically significant higher among male smokers who had exposure to anti-smoking message from 3 channels or 
Table 4. Logistic Regression Analyses of Association between Attempt to Quit Tobacco Smoking with Individual Factors among Male Smokers Aged 15 and Above, in Viet Nam in 2015

\begin{tabular}{|c|c|c|}
\hline Variables & OR & $95 \% \mathrm{CI}$ \\
\hline \multicolumn{3}{|l|}{ Age group } \\
\hline \multicolumn{3}{|l|}{$<=24$} \\
\hline $25-34$ & 1.18 & $0.72-1.92$ \\
\hline $35-44$ & 1.36 & $0.79-2.35$ \\
\hline $45-54 *$ & 1.89 & $1.02-3.51$ \\
\hline$>=55^{*}$ & 2.84 & $1.43-5.66$ \\
\hline \multicolumn{3}{|l|}{ Educational level } \\
\hline \multicolumn{3}{|l|}{ Below primary school } \\
\hline Primary and basic secondary schools & 1.20 & $0.86-1.67$ \\
\hline Secondary school & 0.80 & $0.51-1.25$ \\
\hline Above secondary school & 1.58 & $0.94-2.66$ \\
\hline \multicolumn{3}{|l|}{ Occupation } \\
\hline \multicolumn{3}{|l|}{ Employee } \\
\hline Self-employee & 0.99 & $0.69-1.42$ \\
\hline Unemployed & 1.12 & $0.61-2.07$ \\
\hline Student, Homemaker, Retired, others & 1.01 & $0.55-1.85$ \\
\hline \multicolumn{3}{|l|}{ Wealth } \\
\hline \multicolumn{3}{|l|}{ Poorest } \\
\hline Second poorest & 1.33 & $0.94-1.88$ \\
\hline Averaged & 1.18 & $0.79-1.77$ \\
\hline Second richest & 1.33 & 0.88-1.99 \\
\hline Richest & 0.73 & $0.46-1.18$ \\
\hline \multicolumn{3}{|l|}{ Knowledge of harmful effects on health } \\
\hline \multicolumn{3}{|l|}{$<=3$ diseases } \\
\hline 4-5 diseases & 0.95 & $0.72-1.25$ \\
\hline 6-7 diseases* & 1.97 & $1.45-2.66$ \\
\hline \multicolumn{3}{|l|}{ Residency } \\
\hline \multicolumn{3}{|l|}{ Urban } \\
\hline Rural & 1.08 & $0.84-1.4$ \\
\hline \multicolumn{3}{|l|}{ No. of channels for anti-smoking message } \\
\hline \multicolumn{3}{|l|}{ None } \\
\hline $1-2$ channels & 1.31 & $0.95-1.8$ \\
\hline 3 channels and more* & 1.72 & $1.21-2.45$ \\
\hline \multicolumn{3}{|l|}{ Notice of health warning } \\
\hline \multicolumn{3}{|l|}{ No } \\
\hline Yes & 1.51 & $0.98-2.33$ \\
\hline \multicolumn{3}{|l|}{ Advice to quit by health staff } \\
\hline \multicolumn{3}{|l|}{ No } \\
\hline Yes & 1.91 & $0.74-4.93$ \\
\hline \multicolumn{3}{|l|}{ Number of cigarettes per week } \\
\hline \multicolumn{3}{|l|}{ Less than 70} \\
\hline From 70 to less than 140 & 1.06 & $0.76-1.48$ \\
\hline 140 and more & 0.97 & $0.68-1.4$ \\
\hline \multicolumn{3}{|l|}{ Number of years smoking } \\
\hline \multicolumn{3}{|l|}{ Less than 15 years } \\
\hline $15-24$ years & 0.82 & $0.54-1.26$ \\
\hline $25-34$ years * & 0.59 & $0.36-0.97$ \\
\hline 35 years and more * & 0.40 & $0.22-0.71$ \\
\hline
\end{tabular}

Table 4. Continued

\begin{tabular}{lcc}
\hline Variables & OR & 95\% CI \\
\hline Time after waking up for the first cigarette & & \\
Less than 30 minutes & & \\
30-less than 60 minutes* & 1.29 & $0.92-1.8$ \\
60 minutes and longer * & 1.65 & $1.16-2.34$ \\
$\quad$ Non daily smokers & 1.7 & $1.05-2.74$ \\
\hline *, denotes a statistical significant result & &
\end{tabular}

more (OR=1.72; 95\% CI: 1.21-2.45). Compared to those smokers with less than 15 years smoking, the odds of attempt to quit smoking among smokers who had 25 to 34 years smoking and more than 35 years smoking were only 0.59 and 0.40 times, respectively. Compared to those who needed the first cigarette within 30 minutes after waking up, those who delayed smoking of the first cigarette after 60 minutes and longer were 1.65 times significantly more likely to attempt to quit (OR=1.65; 95\% CI: 1.16-2.34); non daily smokers were 1.7 times more likely to attempt to quit (OR=1.70; 95\% CI: $1.05-2.74)$.

\section{Discussion}

In our study, the proportion of male smokers who attempted to quit tobacco smoking during 12 months prior to the interview was $37.1 \%$. This is in accordance with results reported from Poland for the survey in 2010 (Dorota Kaleta et al., 2012). In China, the survey in found the rate of quit attempt is $25.3 \%$ (Lin Li et al., 2011) . We found that those who had higher level of knowledge on harmful effects of tobacco, who had noticed anti smoking message from more communication channels were significantly more likely to have quit attempts, with the odds ratios ranged from 1.72 (accessed to 3 channels for anti smoking messages or more vs. no channel); to 2.03 (could list 6-7 diseases caused by tobacco use vs. less than 4 diseases). This paper affirms that high level of knowledge of harmful effects of tobacco is predictor for quit attempt as reported in several papers. Study in Poland also indicates the positive relationship between high awareness of smoking health consequences with long-term quitting (Dorota Kaleta et al., 2012) . Survey in China (Lin Li et al., 2011) and in the Australia, Canada, the UK, and the USA (Hyland et al., 2016) among cigarette smokers confirm that negative opinion of smoking is predictor for attempt to quit among smokers. A report based on the Global Adult Tobacco Surveys in 17 countries reported that antismoking information in mass media channel, especially in multiple channels, significantly increased intent to quit smoking with the adjusted odds ratios ranging from 1.3 to 3.2 (Centers for Disease Control and Prevention, 2013).

Although the proportion of smokers who were advised to quit smoking were significantly higher in 2015 as compared to that in 2010 (Ministry of Health of Viet Nam et al., 2010), the rate of quit attempt among male smokers in this survey of 2015 was lower than what were reported in the report of Global Adult Tobacco Survey in 2010 $(55.3 \%)$ (Ministry of Health of Viet Nam et al., 2010). Our 
study could not support for the association between advice to quit by health staff and quit attempt among smokers. A study in Poland also supports this. The study found that $77 \%$ of smokers attempted to quit smoking on their own and only $11 \%$ of smokers who attempted to quit smoking because of physician's investigation, and $41 \%$ of smokers prompted to quit by a personal health problems failed to see the link between their illness and tobacco smoking (Alicja et al., 2008). Roy by his review in 2017 indicates that smoking is a voluntary behavior that can be changed if smokers really want to or motivation appeared to promote them to stop (Roy, 2017). Thus, deeper researches about contents and effectiveness of quit advice by health staff should be conducted to propose appropriate solutions for more efficient advice by health staff. In addition, no improvement in the rate of quit attempt might be explained by the fact that access to anti smoking messages in 2015 did not increase as compared to that in 2010 (Ministry of Health of Viet Nam et al., 2010, 2016). Moreover, sources of anti smoking messages have not been changed in accordance with the trend in information sources preferred by the population, especially in younger generation. During the past few years, most of anti smoking messages have been presented in television and radio programs, while $58 \%$ of the population used less television than before (Vietnam Market Research, 2019). Furthermore, recently social media has become more and more popular, but anti smoking message has not been presented in these channels. According to statistics, the rate of using Internet in Vietnam increased from $30.7 \%$ in 2010 to $52 \%$ in 2016 (International Telecommunication Union (ITU) et al., 2019) and in 2018 among those using internet, 96\% used YouTube, 95\% used Facebook and more than 50\% used Instagram, etc. (The Statistics Portal, 2019). Thus, in order to increase accessibility to anti smoking sources among population, communication programs through social media for anti smoking message are highly recommended (Vietnam Tobacco Control Fund, 2015). Smoking cessation program for young adults through Facebook are proved to be effective for quit attempt, reducing tobacco consumption and stop smoking (Danielle et al., 2015). Our findings support the previous studies indicating that the lower rate of quit attempt are observed among the smokers with higher dependent level (shorter time of the first cigarette after waking up) and with more years of smoking (Greenhalgh et al., 2016; Layoun et al., 2017).

Our survey found statistical significant difference between age groups and that is in line with studies in other countries. Survey in China found that attempt to quit increased with increasing age (Lin Li et al., 2011) and that is in contrast with findings from the prospective cohort survey conducted in the Australia, Canada, the UK, and the USA among cigarette smokers that found that younger age were the factors predictive of making a quit attempt included intention to quit, making a quit attempt in the previous year (Hyland et al., 2016). We did not find any significant discrepancies with regards to quit attempt between occupations, and between wealth level groups. Possible reason for that could be no difference in accessibility and availability of information sources and cessation services between these groups in Vietnam because during the study period, anti-smoking messages were mostly communicated through common media channels and cessation services had not been developed yet.

In conclusion, intervention to improve knowledge of tobacco harmful effects and antismoking communication by multiple and modern communication channels would be effective to raise quit attempt among smokers. Research to understand and promote effectiveness of quit advice by health staff on quit attempt and smoking cessation should be paid more attention.

\section{Author Contribution Statement}

All authors contributed to the manuscript. The first author is responsible for data analysis and making draft of the manuscript. All authors participated in adapting and testing the survey form that is original from and monitoring data collection process, commenting on data analysis and writing, completing the manuscript.

\section{Acknowledgements}

\section{Funding statement}

This study was funded by the Bloomberg Philanthropies. We highly appreciate the contributions to the success of the survey made by the Centers for Disease Control and Prevention in Atlanta, the CDC Foundation, the World Health Organization, the General Statistics Office of Vietnam, and Hanoi Medical University.

If it was approved by any scientific Body/ if it is part of an approved student thesis

The survey was approved by the Ministry of Health and the Hanoi Medical University. The objectives of the survey were clearly explained to the respondents and verbal consents were obtained.

How the ethical issue was handled (name the ethical committee that approved the research)

the Ministry of Health and the Hanoi Medical University

\section{References}

Alicja S, Krzysztof Buczkowski,et al (2008). Patterns of motivations and ways of quitting smoking among Polish smokers: A questionnaire study. BMC Public Health, 8, https://doi.org/10.1186/1471-2458-8-274

Bethany CB, Rachel AS, Megan EP, et al (2016). Transitions in smokers' social networks after quit attempts: A Latent Transition Analysis. Nicotine Tob Res, 18, 2243-51.

Centers for Disease Control and Prevention. (2013). Antismoking Messages and Intention to Quit-17 Countries, 2008-2011. MMWR Morb Mortal Wkly Rep, 62, 417-22.

Danielle ER, Johannes T, Kathryn C, et al (2015). Feasibility and quit rates of the tobacco status project: A Facebook Smoking Cessation Intervention for Young Adults. $J$ Med Internet Res, 17, e291.

Department of Health and Human Services (2020). Smoking Cessation. A Report of the Surgeon General. Department of Health and Human Services, Centers for Disease Control and 
Prevention, National Center for Chronic Disease Prevention and Health Promotion, Office on Smoking and Health.

Dorota K, Przemysław K, Teresa MD, et al (2012). Predictors of long-term smoking cessation: Results from the global adult tobacco survey in Poland (2009-2010). BMC Public Health, 12.

Eleni V, John S, Eline SS, Ron B, Robert W (2011). Predictors of attempts to stop smoking and their success in adult general population samples: A systematic review. Addiction, 106, doi:10.1111/j.1360-0443.2011.03565.x.

Greenhalgh EM, Stillman S, Ford C (2016). 7.7 Factors that predict success or failure in quit attempts. In Scollo, MM and Winstanley, MH [editors] (Tobacco in Australia: Facts and Issues). Melbourne: Cancer Council Victoria. Available from http://www.tobaccoinaustralia.org.au/7-7-personalfactors-associated-with-quitting.

Hitchman SC, Fong GT, Zanna MP, Thrasher JF, Laux FL (2014). The relation between number of smoking friends, and quit intentions, attempts, and success: Findings from the International Tobacco Control (ITC) Four Country Survey. Psychol Addict Behav, 28, 1144-52.

Hyland A, Borland R, Q Li, et al (2016). Individual-level predictors of cessation behaviours among participants in the International Tobacco Control (ITC) Four Country Survey. Tob Control, 15, 83-94.

International Telecommunication Union (ITU), World Bank, \& and United Nations Population Division. (2019). Vietnam internet users.

Layoun N, Hallit S, Waked M, et al (2017). Predictors of past quit attempts and duration of abstinence among cigarette smokers. J Epidemiol Global Health, 7, 199-206.

Lin L, Guoze F, Yuan J, et al (2011). Prospective predictors of quitting behaviours among adult smokers in six cities in China: Findings from the International Tobacco Control (ITC) China Survey. Addiction, 106, 1335-45.

Medawela RMSHB, Ratnayake DRDL, Premathilake LKN, Jayasinghe RD (2021). Attitudes, confidence in practices and perceived barriers towards the promotion of tobacco cessation among clinical dental undergraduates in Sri Lanka. Asian Pac J Cancer Care, 6, 175-9.

Ministry of Health of Viet Nam, Hanoi Medical University, \& General Statistics Office (2010). Global Adult Tobacco Survey (GATS) Viet Nam 2010.

Ministry of Health of Viet Nam, Hanoi Medical University, General Statistics Office of Vietnam, World Health Organization, \& Center for Diseases Control and Prevention in USA (2016). Global Adult Tobacco Survey (GATS): Vietnam 2015. http://www.who.int/tobacco/surveillance/ survey/gats/vietnam-country-report-2015.pdf?ua=1.

National Assembly of Vietnam (2012). Law No 09/2012/QH13: Law on Prevention and Control of Tobacco Harms.

Roy F.B. (2017). Addiction, cigarette smoking, and voluntary control of action: Do cigarette smokers lose their free will? Addictive Behaviors Reports, 5, 67-84.

Stephen Babb, Ann Malarcher, Gillian Schauer, Kat Asman, \& Jamal., A (2017). Quitting Smoking Among Adults-United States, 2000-2015. Morbidity and Mortality Weekly Report, 65, 1457-64.

The Statistics Portal (2019). Leading active social media platforms among internet users in Vietnam in the 2nd and the $3^{\text {rd }}$ quarter of 2018.

Vietnam Market Research (2019). Watching TV behavior of Vietnamese (Vietnamese: Thoi quen xem ti vi cua nguoi Viet).

Vietnam Tobacco Control Fund (2015). Report on Tobacco Control Fund activities (In Vietnamese: Bao cao hoat dong cua Quy Phong chong Tac hai Thuoc la).
World Health Organization (2008). WHO Report on The Global Tobacco Epidemic, 2008- The MPOWER package. World Health Organization.

World Health Organization (2011). WHO Report on The Global Tobacco Epidemic, 2011: Warning about the dangers of tobacco. World Health Organization.

World Health Organization. (2013). Global action plan for the prevention and control of noncommunicable diseases 20132020. World Health Organization.

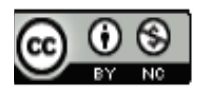

This work is licensed under a Creative Commons AttributionNon Commercial 4.0 International License. 